\title{
Clinical Characteristics and Serotype Distribution of Campylobacter jejuni and Campylobacter coli Isolated from Diarrhoeic Patients in Dhaka, Bangladesh, and Cape Town, South Africa
}

\author{
Khorshed Alam ${ }^{1}$, Albert J Lastovica², Elza le Roux², MAnowar Hossain ${ }^{1 *}$, M Nazrul Islam , Sunil Kumar Sen ${ }^{1}$, \\ Gour C Sur ${ }^{1}$, G Balakrish Nair ${ }^{1}$ and David A Sack ${ }^{1}$ \\ ${ }^{1}$ Laboratory Sciences Division, Centre for Health \& Population Research, International Centre for Diarrhoeal Disease Research, Bangladesh \\ (ICDDR,B), Mohakhali, Dhaka 1212, Bangladesh, ${ }^{2}$ Department of Laboratory Science, Division of Microbiology, Medical School, University of Cape \\ Town, Anzio Road, Observatory, 7925, Cape Town, South Africa
}

[Received 10 August 2006; Accepted 07 October 2006]

\begin{abstract}
Clinical characteristics and serotype distribution of Campylobacter jejuni and Campylobacter coli isolated from paediatric diarrhoeic patients over a three-year period in two geographically diverse areas, Dhaka, Bangladesh, and Cape Town, South Africa, were compared. Both Dhaka and Cape Town patients had similar rates of diarrhoea, vomiting and fever. However, the Dhaka patients were younger (11.8 vs. 13.0 months), had more boys than girls infected (male/female ratio $1.78: 1$ vs. 1.25:1), had more predisposing conditions (26\% vs. 15\%) and had more additional stool pathogens co-isolated with $C$. jejuni/coli than the Cape Town patients. While some $C$. jejuni serotypes were common to both areas, i.e., $0: 4$, other serotypes were present in one location, but not the other. Differences in clinical presentations and serotype distribution in Dhaka and Cape Town are suggestive of different reservoirs for Campylobacter, and different patterns of infection.
\end{abstract}

Keywords: Campylobacter jejuni, Campylobacter coli, Serotyping, Diarrhoea

\section{Introduction}

Campylobacter species are universally acknowledged as the most frequently isolated bacterial pathogens associated with human gastroenteritis, particularly in young children ${ }^{1}$. Campylobacter has also been associated with other clinical conditions such as bacteraemia ${ }^{2-3}$, the Guillain-Barré syndrome ${ }^{4}$, reactive arthritis and pancreatitis $^{1}$.

Most epidemiological investigations of Campylobacter infection have been done in developed countries, and the distribution, epidemiology and clinical relevance of Campylobacter species are not fully understood in developing countries. The aim of this study was to compare clinical details and the serotype distribution of $C$. jejuni and $C$. coli isolates recovered from diarrhoea patients in two geographically diverse areas with a high incidence of paediatric diarrhoea, Dhaka, Bangladesh, and Cape Town, South Africa.

\section{Materials and Methods}

This study, conducted in 2000-2002, included 7,128 diarrhoea patients of all age groups admitted to the Dhaka Hospital of the ICDDR,B: Centre for Health and Population Research. During this same time period, 5,635 diarrhoea patients were admitted to the Red Cross Children's Hospital in Cape Town, South Africa.

In the Dhaka laboratory, Campylobacter organisms were isolated from stools by inoculation onto culture plates containing Brucella selective agar (SR83, Oxoid, Basingstoke, UK), containing 5\% lysed sheep blood and antimicrobial agents including vancomycin (10 mg/l), trimethoprim (5 mg/l), polymyxin B (2,500 U/l), ampotericin B (2 mg/l) and cephalothin (15 mg/l). Culture plates were incubated under microaerobic conditions at $42^{\circ} \mathrm{C}$ in a candle jar and examined $48 \mathrm{~h}$ later. Culture plates considered negative were re-incubated for a further $24 \mathrm{~h}$. Campylobacter isolates were identified by following standard methods ${ }^{5}$. Isolates were preserved in tryptic soy broth (BBL, New Delhi) plus 25\% glycerol and stored at $-80^{\circ} \mathrm{C}$, and subsequently serotyped by the soluble heatstable scheme of Penner ${ }^{6}$. The Cape Town laboratory supplied the 60 different reference antisera used for serotyping.

In the Cape Town laboratory, Campylobacter, Arcobacter or Helicobacter isolates were recovered from diarrhoeic stools by the "Cape Town" protocol", which employs membrane filtration onto antibiotic-free tryptose blood agar (CM 233, Oxoid, Bassingstoke, UK) containing $10 \%$ un-lysed horse blood, and incubation at $37^{\circ} \mathrm{C}$

*Corresponding author:

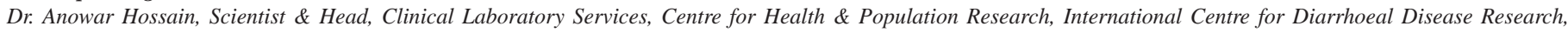
Bangladesh (ICDDR,B), GPO Box 128, Mohakhali, Dhaka 1212, Bangladesh

Tel (Office): (02) 8826391, (02) 8860523-32; Tel (Home): 8856767, 8833590; Cell: 0171 3093855; Fax: +880 (02) 8812529; E-mail: anowar@icddrb.org 
in an $\mathrm{H}_{2}$-enriched microaerobic atmosphere (BR38, Oxoid) for up to 6 days. Isolates were characterized as $C$. jejuni or $C$. coli on the basis of established tests ${ }^{1,5}$. Serotyping was done with antisera raised against 60 reference bacteria of the Penner scheme ${ }^{6}$.

\section{Results and Discussion}

Of the 7,128 diarrhoeic stool specimens examined in the Dhaka laboratory, 604 (8.5\%) were positive for Campylobacter jejuni and Campylobacter coli. One hundred and eighty one (30\%) of the Campylobacter isolates were randomly selected for clinical assessment and serotyping. Ages of the patients ranged from 1 month to 60 years, and 115 (64\%) of the 181 patients were less than 6 years old (Table 1). The male/female ratio was $1.78: 1.00$. Almost all (96\%) of the Dhaka patients had diarrhoea, 10\% had vomiting and $7 \%$ had fewer. For $50 \%$ of the stools from patients in Dhaka, Campylobacter was the sole pathogen isolated. In the remaining stools, Campylobacter was co-isolated with one or more of isolates of Vibrio, Shigella, Salmonella, Aeromonas, Plesiomonas, or Hafnia. Most Campylobacter isolations occurred in March to May, the hottest season of the year, and preceding the annual monsoon season.

Table 1. Clinical characteristics of patients with Campylobacter jejuni or Campylobacter coli infection in Dhaka, Bangladesh and Cape Town, South Africa

\begin{tabular}{|c|c|c|}
\hline Feature & $\begin{array}{c}\text { Dhaka, } \\
\text { Bangladesh }\end{array}$ & $\begin{array}{l}\text { Cape Town, } \\
\text { South Africa }\end{array}$ \\
\hline No. of patients & 181 & 348 \\
\hline Age range & 1 month - 60 years & 1 month - 12 years \\
\hline Male/female ratio & $1.78 / 1.00$ & $1.25 / 1.00$ \\
\hline $\begin{array}{l}<2 \text {-year-old \% } \\
\text { (mean age) }\end{array}$ & 50.3 (11.8 months) & 65.1 (13.0 months) \\
\hline $\begin{array}{l}\text { Campylobacter as } \\
\text { sole pathogen (\%) }\end{array}$ & 50.0 & 89.0 \\
\hline $\begin{array}{l}\text { Stool characteristics (\%) } \\
\text { (watery, loose, } \\
\text { dysenteric) }^{*}\end{array}$ & $95.5,0.0,4.5$ & 10.6, 78.2, 11.2 \\
\hline $\begin{array}{l}\text { Diarrhoea, Vomiting, } \\
\text { Fever }>38^{\circ} \mathrm{C}(\%)\end{array}$ & $96.0,10.0,7.0$ & $93.0,8.0,5.0$ \\
\hline $\begin{array}{l}\text { Pre-existing clinical } \\
\text { conditions }(\%)^{* *}\end{array}$ & 26.0 & 15.0 \\
\hline
\end{tabular}

Of the 5,635 diarrhoea stool specimens examined in the Cape Town laboratory, 999 (17.8\%) were positive for 10 species of Campylobacter, 2 species of Helicobacter and Arcobacter butzleri. Of these 999 isolates, 316 were identified as C. jejuni and 32 as C. coli that formed $34.8 \%$ (348/999) of the total campylobacteracae or helicobacteraceae isolated (Table 2). These 348 C. jejuni or C. coli isolates were selected for clinical assessment and serotyping ${ }^{6}$, for a direct comparison with the
C. jejuni and C. coli isolates recovered in Dhaka. The Penner serotyping system is only effective in typing $C$. jejuni, C. coli or C. lari, and not other species of Campylobacter, Helicobacter, or Arcobacter. Patient ages ranged from 1 month to 12 years of age. Sixty-five percent of the Cape Town patients were 2 years old or less, and the male/female ratio was 1.25:1.00.

Table 2. Distribution of Campylobacter species and related microorganisms isolated from 5,635 diarrhoeic stools at the Red Cross Children's Hospital, Cape Town, South Africa, 2000-2002

\begin{tabular}{lc}
\hline Bacterium & Isolation rate, No. (\%) \\
\hline $\begin{array}{l}\text { Campylobacter jejuni subsp. jejuni } \\
\text { biotypes } 1 \& 2^{*}\end{array}$ & $316(31.6)$ \\
Campylobacter concisus & $234(23.4)$ \\
Campylobacter upsaliensis & $226(22.6)$ \\
Campylobacter jejuni subsp. doylei & $91(9.1)$ \\
Helicobacter fennelliae & $64(6.4)$ \\
Campylobacter coli & $32(3.2)$ \\
Campylobacter hyointestinalis & $13(1.3)$ \\
Helicobacter cinaedi & $10(1.0)$ \\
CLO/HLO $\dagger$ & $5(0.5)$ \\
Arcobacter butzleri & $4(0.4)$ \\
Campylobacter fetus subsp. fetus & $1(0.1)$ \\
Campylobacter lari & $1(0.1)$ \\
Campylobacter curvus & $1(0.1)$ \\
Campylobacter sputorum biovar. sputorum & $1(0.1)$ \\
\hline Total & $999(100.0)$ \\
\hline * Bompylyping scheme of Skirrow and Benjamin 5 ; $\dagger=$ CLO/HLO: \\
characterized.
\end{tabular}

In $89 \%$ of the Cape Town patients with campylobacteriosis, Campylobacter was the sole pathogen isolated, while in the remaining stool samples, Campylobacter were co-isolated with Salmonella, or Shigella. Most Cape Town patients with C. jejuni or C. coli infection had diarrhoea (93\%), 8\% were vomiting and (5\%) had fever $>38^{\circ} \mathrm{C}$. More than $11 \%$ of the patients had dysentery.

Fifteen percent of the South African patients had pre-existing clinical conditions such as, anaemia, respiratory diseases, HIV+ status and malnutrition (Table 1). The majority of Campylobacter isolations occurred during December to March, which is the late summer/early autumn in South Africa, and a pattern that is reflective of developed countries ${ }^{8}$.

Of the 181 isolates serotyped in Dhaka, 112 isolates reacted with the reference antisera. Eighty-two (45.3\%) isoaltes reacted with a single serotype, 30 (16.6\%) reacted with multiple (2 or more), serotypes, and 69 (38.1\%) were non-typable. Among the 82 single serotype isolates, 26 different serotypes were identified; serotype O:33 was the most frequent, followed by serotypes O:22, O:3, 
O:55, O:26 and O:1. Among the isolates with multi-antigenic expression, 36 different serotypes were noted; serotype O:23/ O:36 was the most common, followed by serotype O:1/O: 8 (Table 3). One isolate typed as serotype O:41, which has been recognized to be associated with the Guillain-Barré Syndrome in South Africa and elsewhere ${ }^{4}$.

Table 3. Serotype distribution of Campylobacter jejuni isolated from diarrhoeic patients in Dhaka, Bangladesh and Cape Town, South Africa

\begin{tabular}{|c|c|c|}
\hline Serotype & Isolate No. & Frequency (\%) \\
\hline \multicolumn{3}{|c|}{ Dhaka, Bangladesh $(\mathrm{n}=181)$} \\
\hline O:33 & 12 & 6.6 \\
\hline $\mathrm{O}: 22$ & 8 & 4.4 \\
\hline O:55 & 7 & 3.8 \\
\hline O:1 & 6 & 3.3 \\
\hline $\mathrm{O}: 3$ & 6 & 3.3 \\
\hline O:26 & 6 & 3.3 \\
\hline $0: 42$ & 5 & 2.8 \\
\hline $0: 53$ & 4 & 2.2 \\
\hline $\mathrm{O}: 40$ & 4 & 2.2 \\
\hline $\mathrm{O}: 4$ & 1 & 0.5 \\
\hline O:41 & 1 & 0.5 \\
\hline NT & 69 & 38.1 \\
\hline Multi-reactive* $^{*}$ & 30 & 16.6 \\
\hline Other $^{* *}$ & 23 & 12.9 \\
\hline \multicolumn{3}{|c|}{ Cape Town, South Africa $(\mathrm{n}=316)$} \\
\hline $\mathrm{O}: 2$ & 18 & 5.8 \\
\hline O:1 & 13 & 4.2 \\
\hline $\mathrm{O}: 4$ & 10 & 3.5 \\
\hline O:37 & 9 & 2.9 \\
\hline O:5 & 8 & 2.6 \\
\hline O:12 & 8 & 2.6 \\
\hline $\mathrm{O}: 22$ & 7 & 2.2 \\
\hline O:33 & 1 & 0.3 \\
\hline $\mathrm{O}: 3$ & 1 & 0.3 \\
\hline NT & 56 & 17.9 \\
\hline Multi-reactive* & 70 & 22.4 \\
\hline Other ${ }^{* * *}$ & 112 & 35.6 \\
\hline
\end{tabular}

NT $=$ Non-typable; ${ }^{*}=$ Reacts in two or more antisera; Other $^{* *}=$ Reacts in 15 additional antisera; Other ${ }^{* * *}=$ Reacts in 21 additional antisera

These results contrast with the serotyped Cape Town C. jejuni isolates, among which $18 \%$ were non-typable and $22 \%$ reacted with two or more antisera. Antigenic extracts of $60 \%$ of the Cape Town C. jejuni isolates reacted with only one test antisera, and the most frequent serotype detected was $\mathrm{O}: 2$, followed by $\mathrm{O}: 1, \mathrm{O}: 4$ and O:37 (Table 3). Non-typable isolates predominated for C. coli isolates from both Cape Town and Dhaka, and the remaining C. coli isolates represented a variety different serotypes (Table 4).
Table 4. Serotype distribution of Campylobacter coli isolates recovered from diarrhoeic patients in Dhaka, Bangladesh and Cape Town, South Africa

\begin{tabular}{lcc}
\hline Serotype & Isolate No. & Frequency $(\%)$ \\
\hline Dhaka, Bangladesh $(\mathrm{n}=15)$ & 12 & 80.0 \\
NT & 1 & 6.6 \\
O:20/O:26 & 1 & 6.6 \\
O:28/O:55 & 1 & 6.6 \\
O:64 & & \\
Cape Town, South Africa $(\mathrm{n}=32)$ & 6 & 18.7 \\
NT & 4 & 12.5 \\
O:30 & 4 & 12.5 \\
O:46 & 3 & 9.4 \\
O:49 & 3 & 9.4 \\
O:5 & 2 & 6.3 \\
O:5/O:30 & 2 & 6.3 \\
O:20 & 2 & 6.3 \\
O:24 & 1 & 3.1 \\
O:26/O:34 & 1 & 3.1 \\
O:34 & 1 & 3.1 \\
O:24/O:30 & 1 & 3.1 \\
O:26 & 1 & 3.1 \\
O:66 & 1 & 3.1 \\
O:54 & 1 & \\
\hline NT $=$ Non-typable & & \\
\hline
\end{tabular}

NT = Non-typable

Our data indicates a high level of diversity among serotyped isolates of $C$. jejuni and $C$. coli isolated from diarrhoeic patients in both Dhaka and Cape Town. The high incidence of non-typable isolates in Dhaka implies that these isolates have antigenic specificities, which are undetectable by the current serotyping system. Some serotypes were found in one study area but not in the other (serotype 0:55 in Dhaka, serotype O:2 in Cape Town). This could be due to different animal reservoirs for Campylobacter in these areas, e.g., pigs are much more commonly consumed in Cape Town than Bangladesh. Serotype 0:2 was not detected in Dhaka in this study as well as not in a previous one ${ }^{9}$, but it was the most commonly detected serotype (5.8\%) in the Cape Town. Serotype 0:2 is one of the most commonly and consistently detected serotypes world-wide ${ }^{10-11}$. Antigenic variability (i.e., serotypes 0:55 in Dhaka, and O:2 in Cape Town) indicates persistence over time of virulent $C$. jejuni clones in a confined geographical area.

The clinical and serological observations of patients in Dhaka and Cape Town indicated both similarities and differences. Our observations suggest dissimilarity in patterns of infection, and the prevalence and persistence in environmental and animal reservoirs of Campylobacter between these two population groups. One of the Bangladeshi $C$. jejuni isolates was serotyped as O:41. This serotype is known to be associated with the GuillainBarré syndrome in patients in Cape Town and elsewhere ${ }^{4}$, but the true incidence and causative agents of the Guillain-Barré syndrome in Bangladesh remains unknown at present. Additional 
epidemiological, serological and molecular studies are essential for a more complete understanding of the disease potential and other aspects of Campylobacter infection in our patients.

Different isolation protocols in the two laboratories yielded different Campylobacter prevalence rates. With the candle jar method used in Dhaka, only C. jejuni and C. coli were isolated, while in Cape Town, the "Cape Town" protocol ${ }^{7}$ yielded isolates of 10 Campylobacter species, 2 Helicobacter species and Arcobacter butzleri. Many of these species are seldom isolated elsewhere and their true disease is largely unknown. Possibly altering the isolation protocol in Dhaka could be more efficient for the isolation of all Campylobacter species and provide a better appreciation of the disease potential of all Campylobacter species.

In both Cape Town and Dhaka the high diversity of serotypes recognized is suggestive of multiple source of infections. This high rate of infection with multiple pathogens could indicate Campylobacter transmission from reservoirs with little seasonal variation, such as infected chicken, rather than contamination of previously uninfected food sources which may be enhanced at higher temperatures ${ }^{8}$.

In 1987, Neogi and Shahid ${ }^{9}$ documented the serotype distribution of 102 C. jejuni isolates isolated from diarrhoeic patients attending the ICDDR,B in Bangladesh. C. jejuni was recovered from $7 \%$ of the patients tested. Of the Campylobacter isolates examined, 74\% were typable, and serotypes O:53, O:15 and O:22 predominated ${ }^{9}$. Our current results indicated that $62 \%$ of the Bangladeshi C. jejuni isolates tested was typable. This high incidence of non-typable isolates (38\%) in Dhaka implies these isolates have antigenic specificities unable to be detected by the current serotyping system. While serotypes O:53 and O:22 predominated in our study, serotype 0:15 was not detected (Table 3). The persistence of serotypes O:22 and O:53 in Dhaka over a 17-year period may indicate a stable reservoir in Dhaka for Campylobacter isolates of these antigenic specificities.

\section{Acknowledgements}

This study was funded by the ICDDR, B: Centre for Health and Population Research and its donors who provide unrestricted support to the Centre for its operations and research. Current donors providing unrestricted support include: Australian International Development Agency (AusAID), Canadian International Development Agency (CIDA), Department for
International Development, UK (DFID), Government of Bangladesh, Government of Japan, Government of Sri Lanka, Government of the Netherlands, Swedish International Development Cooperative Agency (SIDA), the Kingdom of Saudi Arabia and Swiss Development Cooperation (SDC). We gratefully acknowledge these donors for their support and commitment to the Centre's research efforts. AJL is indebted to the University of Cape Town and the South African Medical Research Council for financial support.

\section{References}

1. Lastovica AJ \& Skirrow MB. 2000. Clinical significance of Campylobacter and related species other than Campylobacter jejuni and C. coli. In Campylobacter (Nachamkin I \& Blaser MB eds), $2^{\text {nd }}$ edn, pp 89-120. ASM Press, Washington DC.

2. Lastovica AJ. 1996. Campylobacter/Helicobacter bacteraemia in Cape Town, South Africa, 1977-1995. In Campylobacters, Helicobacters and Related Organisms. (Newell D, Ketley JM \& Feldman RA eds), pp 475-479. Plenum Press, New York.

3. Lastovica AJ, Goddard EA \& Argent AC. 1997. Guillain-Barré syndrome in South Africa associated with Campylobacter jejuni O:41 strains. J Infect Dis. 176 (Suppl): S139-S143.

4. Skirrow MB \& Benjamin J. 1980. “1001” Campylobacters from man and animals. J Hyg. 85: 427-442.

5. Penner JL, Hennessey JN \& Congi RV. 1983. Serotyping of Campylobacter jejuni and Campylobacter coli on the basis of thermostable antigens. Eur J Clin Microbiol. 2: 378-383.

6. Le Roux E \& Lastovica AJ. 1998. The Cape Town Protocol: How to isolate the most campylobacters for your dollar, pound, franc, yen, etc. In Campylobacter, Helicobacter \& Related Organisms (Lastovica AJ, Newell DG \& Lastovica EE. eds), pp 30-33. University of Cape Town, Cape Town.

7. Oberhelman RA \& Taylor DA 2000. Campylobacter infections in developing countries. In Campylobacter (Nachamkin I \& Blaser MB eds), $2^{\text {nd }}$ edn, pp 139-153. ASM Press, Washington DC.

8. Neogi PK \& Shahid NS. 1987. Serotypes of Campylobacter jejuni isolated from patients attending a diarrhoeal disease hospital in urban Bangladesh. J Med Microbiol. 24: 303-307.

9. Chatzipanagiotou S, Papavasileiou E, Lakumenta A, Makri A, Nicolaou C, Chantzis K, Manganas S \& Legakis N. 2003. Heat-stable antigen serotyping of Campylobacter jejuni strains isolated from hospitalised children in Athens, Greece. Eur J Epidemiol. 18: 10971100.

10. Nielsen EM, Engberg J \& Madsen M. 1997. Distribution of serotypes of Campylobacter jejuni and C. coli from patients, poultry, cattle and swine. FEMS Immun Med Microbiol. 19: 47-56.

11. Wareing DR, Bolton FJ, Fox AJ, Wright PA \& Greenway DL. 2002. Phenotypic diversity of Campylobacter isolates from sporadic cases of human enteritis in the UK. J Appl Microbiol. 92: 502-509. 